\title{
The impact of hyperglycemia and hyperlipidemia on plasma P-selectin and platelet markers after ischemic stroke
}

\author{
Malgorzata Pawelczyk ${ }^{1}$, Beata Kaczorowska ${ }^{1}$, Zbigniew Baj²
}

\begin{abstract}
1Department of Neurology and Stroke, Medical University of Lodz, Lodz, Poland 2Department of Pathophysiology and Clinical Immunology, Medical University of Lodz, Lodz, Poland
\end{abstract}

Submitted: 7 April 2016

Accepted: 21 September 2016

Arch Med Sci 2017; 13, 5: 1049-1056

DOI: https://doi.org/10.5114/aoms.2017.65816

Copyright @ 2017 Termedia \& Banach

\section{Abstract}

Introduction: Platelet activation plays a key role in the pathogenesis of ischemic cerebrovascular diseases. Thus, it is very important to identify novel pharmacological targets for platelet inhibition to improve ischemic stroke treatment. The aim of the study was to assess the relationship between metabolic disorders and platelet activity markers in patients with acute ischemic stroke.

Material and methods: Ninety-four patients with acute ischemic stroke were divided into four groups with: normolipidemia and normoglycemia (NL/NG), $n=25$; normolipidemia and hyperglycemia (NL/HG), $n=21$; hyperlipidemia and normoglycemia (HL/NG), $n=27$; hyperlipidemia and hyperglycemia (NL/NG), $n=21$. Twenty-one healthy subjects served as controls. We assessed the CD62P expression on resting and thrombin-activated blood platelets using the flow cytometer and anti-CD61 and anti-CD62P monoclonal antibodies. CD61-positive microparticles were defined as platelet-derived microparticles. The level of sP-selectin in serum was measured by the ELISA method.

Results: We observed a significant influence of hyperlipidemia and hyperglycemia on sP-selectin concentration. A strong correlation between higher SP-selectin concentration and enhanced LDL $(p=0.001)$, total cholesterol $(p=0.02), \mathrm{HbA}_{1 c}$ level $(p<0.001)$ was noticed. The level of sP-selectin and PDMPs $(p<0.001)$ were significantly higher in all groups of stroke patients compared with the controls. CD62P expression on resting and thrombin activated platelets were significantly lower in groups of patients with stroke. Conclusions: Hyperlipidemia and hyperglycemia exert an equal stimulatory effect on tested platelet markers but with no synergistic action in stroke patients with both of the metabolic comorbidities. sP-selectin concentration in stroke patients best reflects the impact of hyperglycemia and hyperlipidemia on vascular lesions and platelet activation.

Key words: platelet activation, stroke, hyperlipidemia, hyperglycemia, P-selectin, CD61, CD62P.

\section{Introduction}

Blood platelets play a crucial role in the maintenance of normal hemostasis and pathogenesis of hemostatic disorders and atherosclerosis. Platelets' procoagulant properties are associated with procoagulant

\author{
Corresponding author: \\ Malgorzata Pawelczyk \\ Department of Neurology \\ and Stroke \\ Medical University of Lodz \\ 113 Zeromskiego St \\ 90-549 Lodz, Poland \\ Phone: +48 426393591 \\ Fax: +48 426393791 \\ E-mail: malgorzata. \\ pawelczyk@umed.lodz.pl
}


phospholipids, but also with specific protein receptors for the coagulation factors and proteases existing in platelet surface membranes [1-3]. Metabolic disorders, such as diabetes mellitus (DM) and hyperlipidemia, are the key factors in the development of atherosclerosis, platelet activation and other vascular diseases.

Circulating atherogenic lipoproteins have been shown to activate circulating blood platelets, and thereby contribute to the pathogenesis and progression of atherothrombosis. Platelets activated by native and oxidized low-density lipoprotein $(\mathrm{LDL})$ release factors which increase the expression of $L D L$ receptors on macrophages and stimulate LDL accumulation in these cells [4]. This interaction can further contribute to the progression of atherogenesis [5].

Diabetes mellitus is a hypercoagulable state. Several factors contribute to the prothrombotic condition which characterizes patients with DM. The most important of these factors are increased coagulation, impaired fibrinolysis, endothelial dysfunction and platelet hyperreactivity [6-8].

Platelet abnormalities in diabetes mellitus, including altered adhesion and aggregation, are the result of hypersensitivity of diabetic platelets to agonists [9]. Platelet dysfunction in diabetic patients is caused by several mechanisms such as hyperglycemia, insulin deficiency and associated metabolic conditions (obesity, dyslipidemia and enhanced systemic inflammation) [10]. Hyperglycemia may increase platelet reactivity by glycating platelet surface proteins, activating protein kinase $C$ (a mediator of platelet activation) and increasing P-selectin expression. Insulin deficiency and endothelial dysfunction also play an important role in platelet dysfunction; however, platelets in diabetic patients are in an activated state even in the absence of vascular injury [10].

One of the most important markers of platelet activation is the adhesion molecule P-selectin [11]. This adhesion molecule is a component of the platelet $\alpha$-granule membrane and of the Weibel-Palade body membrane of endothelial cells. Platelet and endothelial cell activation results in hyperexpression of P-selectin on the surface of these cells; however, the increased P-selectin expression caused by platelet activation and degranulation is short lasting. Degranulated platelets rapidly shed the surface P-selectin to the plasma pool and P-selectin circulates in plasma in a soluble form (sP-selectin) [12, 13]. The increased plasma concentration of soluble $\mathrm{P}$-selectin reflects the release of this adhesion molecule from activated platelets or damaged endothelial cells. It was recently shown that an elevated concentration of sP-selectin can exert a procoagulant effect [14], and it might play an important role in thrombotic disorders. The increase of sP-selectin concentration is also associated with formation of platelet-derived microparticles (PDMPs). They influence the normal hemostatic response to vascular injury, and also express phospholipids that function as procoagulants [15].

The aim of the study was to investigate the relationship between the metabolic disorders, such as hyperlipidemia and hyperglycemia, and selected platelet activity markers (soluble P-selectin, platelet P-selectin expression and PDMPs) in patients with acute ischemic stroke. This relationships may be an important target for ischemic stroke treatment and prevention.

The study was approved by the Ethics Committee of the Medical University of Lodz, Poland (No. RNN/465/11/KB).

\section{Material and methods}

The study group consisted of 94 patients with acute ischemic stroke ( $\leq 7$ days) hospitalized in the Department of Neurology and Stroke, Medical University of Lodz, Poland. The clinical diagnosis of ischemic stroke was established using the medical history of the patients, neurological investigation and cranial computed tomography. To avoid the influence of stroke subtype on the results, only patients with stroke of large-artery atherosclerosis (LAA), based on the TOAST classification criteria [16], were included in the study. Patients with a history of infection shortly before stroke were excluded to avoid the influence of the coexisting infection on platelet activity markers. Patients with severe liver disease, renal failure, cancer, chronic inflammatory diseases, and hemorrhagic diathesis were not enrolled in the study.

The diagnosis of diabetes mellitus in patients with hyperglycemia was established before the hospitalization and diabetic patients were included in the study if glycated hemoglobin $\left(\mathrm{HbA}_{1}\right)$ was higher than $7 \%$ in spite of hypoglycemic treatment (metformin $n=15$, glimepiride $n=6$, gliclazide $n=8$, gliclazide + metformin $n=4$, insulin therapy $n=9)$. The hyperlipidemia was recognized on the basis of a LDL cholesterol (LDL-C) level $\geq 2.6 \mathrm{mmol} / \mathrm{l}$ $(100 \mathrm{mg} / \mathrm{dl})$; however, all stroke patients received atorvastatin (20 mg/day) during hospitalization.

All studied stroke patients received a $300 \mathrm{mg}$ dose of acetylsalicylic acid (ASA) on the first day, and they continued the treatment with a $75 \mathrm{mg}$ ASA dose during the hospitalization. The patients in the control group received ASA $75 \mathrm{mg} /$ day.

The stroke patients were divided into four groups:

1) 25 patients with normolipidemia and normoglycemia - NL/NG: LDL < $2.6 \mathrm{mmol} / /(100 \mathrm{mg} /$ dl) [17], mean: $2.20 \pm 0.32 \mathrm{mmol} / \mathrm{l} ; \mathrm{HbA}_{1 \mathrm{c}} \leq 7 \%$ [18], mean: $5.7 \pm 0.3 \%$; 11 males, 14 females, mean age: $76.44 \pm 12.6$ years; 
2) 21 patients with normolipidemia and hyperglycemia - NL/HG: LDL < $2.6 \mathrm{mmol} / \mathrm{l}(100 \mathrm{mg} / \mathrm{dl})$, mean: $2.33 \pm 0.34 \mathrm{mmol} / \mathrm{l} ; \mathrm{HbA}_{1 \mathrm{c}}>7 \%$, mean: $7.67 \pm 0.82 \%$; 11 males, 10 females, mean age: $73.1 \pm 8.21$ years;

3) 27 patients with hyperlipidemia and normoglycemia - HL/NG: LDL $\geq 2.6 \mathrm{mmol} / \mathrm{l}(100 \mathrm{mg} / \mathrm{dl})$, mean: $3.23 \pm 1.28 \mathrm{mmol} / \mathrm{l} ; \mathrm{HbA}_{1 \mathrm{c}} \leq 7 \%$, mean: $5.53 \pm 0.33 \%$; 9 males, 18 females, mean age: $66.07 \pm 12.39$ years;

4) 21 patients with hyperlipidemia and hyperglycemia - HL/HG: LDL $\geq 2.6 \mathrm{mmol} / \mathrm{l}(100 \mathrm{mg} / \mathrm{dl})$, mean: $3.26 \pm 0.56 \mathrm{mmol} / \mathrm{l} ; \mathrm{HbA}_{1 \mathrm{c}}>7 \%$, mean: $7.91 \pm 1.16 \%$; 13 males, 8 females, mean age: $70.1 \pm 10.76$ years;

Twenty-one age- and sex-matched control subjects (CS) - LDL $<2.6 \mathrm{mmol} / \mathrm{l}(100 \mathrm{mg} / \mathrm{dl})$, mean: $2.43 \pm 0.13 \mathrm{mmol} / \mathrm{l} ; \mathrm{HbA}_{1 \mathrm{c}} \leq 7 \%$, mean: 5.22 $\pm 0.37 \%$; 10 males, 11 females, mean age: 60.2 \pm 14.6 years - were recruited from patients with discopathy or tension-type headache. Subjects were excluded from the control group if they had a history of cerebrovascular diseases. Otherwise, the exclusion criteria were identical to those applied to the patient groups.

The risk factors for ischemic stroke (arterial hypertension, ischemic heart disease and body mass index) were similar in the study groups and the controls, and patients in all groups received antihypertensive drugs, such as angiotensin convertase enzyme (ACE) inhibitors, calcium channel blockers, and $\beta$-blockers.

Complete blood cell count, $\mathrm{HbA}_{1 c}$, total cholesterol (TC), high-density lipoprotein cholesterol (HDL-C), and LDL-C levels were measured in a fasting state for all patients. Serum LDL-C levels were calculated with Friedewald's formula [19]. Biochemical determinations were performed with the Olympus AU640 Analyzer (Olympus Optical Co., Ltd., Shizuoka, Japan).

The flow cytometry (FACScan, Becton Dickinson, San Jose, USA) was used to measure the expression of P-selectin (CD62P) on resting and thrombin-activated platelets. Blood was withdrawn without stasis to avoid platelet activation and collected into two tubes. The first tube contained $0.1 \mathrm{ml}$ of blood and $1 \mathrm{ml}$ of a $0.5 \%$ solution of paraformaldehyde in PBS - this sample reflected the state of platelet activity ex vivo; the second tube containing $0.5 \mathrm{ml}$ of blood and $0.5 \mathrm{ml}$ of EDTA was used to assess platelet reactivity after treatment with 0.08 units of bovine thrombin for 4 min. All platelet measurements were performed within 90 min after blood withdrawal. The antibody anti-CD61-FITC (Dako) - a fluorescein-isothiocyanate-conjugated antibody to glycoprotein Illa - was used as an activation-independent marker of platelets. A phycoerythrin-conjugated
anti-CD62-PE antibody (Becton Dickinson) was used to assess $\alpha$-granule degranulation. To assess the extent of the nonspecific association of protein with platelets, a control tube containing antiCD61-FITC and nonfractionated PE conjugated IgG (Becton Dickinson) was used for each blood sample. The reaction mixture was incubated in a dark room, at room temperature, for $30 \mathrm{~min}$. Then, the antibody-bound platelets were fixed with $200 \mu \mathrm{l}$ of FACS flow liquid and analyzed.

Platelets were subtracted from other blood cells and identified by flow cytometry based on size and platelet-specific CD61 surface expression. The data were presented as the percentage of $\mathrm{CD} 62 \mathrm{P}(+)$ platelets (\%CD62) and as the median fluorescence intensity (medCD62) reflecting the density of CD62P on the platelet surface. CD61-positive microparticles were defined as platelet-derived microparticles (PDMPs). They were distinguished from other platelets on forward scatter histograms based on their size $<0.2 \mu \mathrm{m}$. WinMDI 2.8 was used to analyze the data collected with flow cytometry.

The level of sP-selectin was estimated by ELISA (Quantikine human sP-selectin/CD62P ELISA Kit, R\&S Systems, Abingdon, UK). After $10 \mathrm{~min}$ of centrifugation, the blood plasma obtained from EDTA-anticoagulated samples was stored at $-80^{\circ} \mathrm{C}$ until measurements. Measurement of sP-selectin was performed according to the manufacturer's instructions.

\section{Statistical analysis}

Since all study variable did not pass the D'Agostino normality test, differences between groups were analyzed using the Kruskal-Wallis test followed by the post-hoc Dunn's test for multiple comparisons. Spearman's correlation was performed to assess the relationship between continuous variables. All of the statistical analyses were performed using Statistica for Windows ver. 8.0. The null hypothesis was rejected if $p<0.05$.

\section{Results}

The blood lipid profile and $\mathrm{HbA}_{1 \mathrm{c}}$ level in study groups are presented in Table I.

In our study we aimed to demonstrate the influence of metabolic disorders (hyperlipidemia and hyperglycemia) on the platelet activity markers. The most interesting finding was the significant positive correlation between LDL, TC, $\mathrm{HbA}_{1 c}$ level and sP-selectin concentration (LDL : sP-selectin $p=0.001$, TC : sP-selectin $p=0.02, \mathrm{HbA}_{1 \mathrm{c}}$ : sP-selectin $p<0.001)$. The strongest positive correlation was observed between sP-selectin concentration and $\mathrm{HbA}_{1 \mathrm{c}}$ level (Figure 1). We also noted significantly higher serum concentration of sP-se- 
Table I. Blood lipid profile and $\mathrm{HbA}_{1 \mathrm{c}}$ level (means $\pm \mathrm{SD}$ ) in study groups

\begin{tabular}{|lccccc|}
\hline Parameters & NL/NG & NL/HG & HL/NG & HL/HG & CS \\
\hline TC $[\mathrm{mmol} / \mathrm{l}]$ & $3.68 \pm 0.6$ & $3.77 \pm 0.45$ & $5.66 \pm 0.9$ & $5.86 \pm 0.46$ & $4.06 \pm 0.5$ \\
\hline LDL $[\mathrm{mmol} / \mathrm{l}]$ & $2.15 \pm 0.33$ & $2.33 \pm 0.34$ & $4.62 \pm 1.27$ & $4.28 \pm 0.56$ & $2.45 \pm 0.13$ \\
\hline $\mathrm{HDL}[\mathrm{mmol} / \mathrm{l}]$ & $1.08 \pm 0.3$ & $1.07 \pm 0.31$ & $1.3 \pm 0.31$ & $1.14 \pm 0.25$ & $0.95 \pm 0.17$ \\
\hline $\mathrm{TG}[\mathrm{mmol} / \mathrm{l}]$ & $1.38 \pm 0.72$ & $1.56 \pm 0.7$ & $1.66 \pm 0.85$ & $1.67 \pm 0.61$ & $1.31 \pm 0.48$ \\
\hline $\mathrm{HbA}_{1 \mathrm{c}}[\%]$ & $5.7 \pm 0.3$ & $7.5 \pm 0.9$ & $5.53 \pm 0.33$ & $7.66 \pm 1.3$ & $5.22 \pm 0.37$ \\
\hline
\end{tabular}

NL/NG - normolipidemic/normoglycemic group, NL/HG - normolipidemic/hyperglycemic group, HL/NG - hyperlipidemic/normoglycemic group, HL/HG - hyperlipidemic/hyperglycemic group, CS - control subjects; SD - standard deviation, TC - total cholesterol, LDL - lowdensity lipoprotein cholesterol, $H D L$ - high-density lipoprotein cholesterol, $T G$ - triglyceride, $H b A_{1 c}-$ glycosylated hemoglobin $A_{1 c^{*}}$
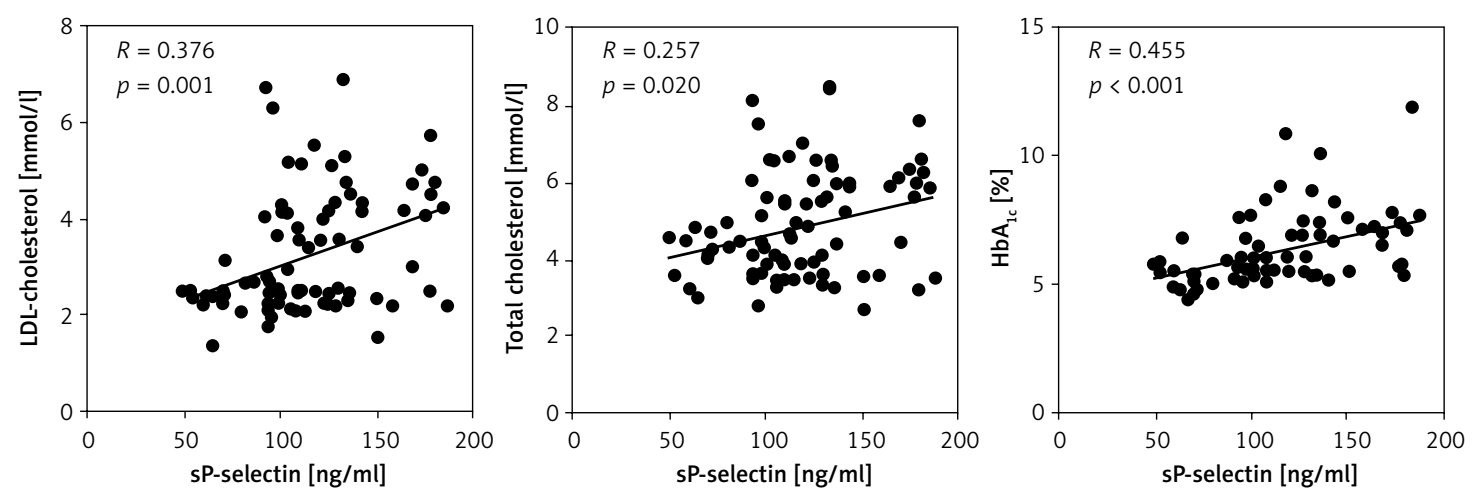

Figure 1. Correlations between SP-selectin concentration and LDL, total cholesterol, and $\mathrm{HbA}_{1 \mathrm{c}}$ level

lectin in all stroke patients compared with the control group (NL/NG vs. CS $p<0.001$ and NL/ $\mathrm{HG}, \mathrm{HL} / \mathrm{NG}, \mathrm{HL} / \mathrm{HG}$ vs. CS $p<0.0001)$. The concentration of sP-selectin was significantly higher in patients with metabolic disorders compared to NL/NG stroke patients (NL/HG vs. NL/NG $p<$ $0.01 ; \mathrm{HL} / \mathrm{NG}, \mathrm{HL} / \mathrm{HG}$ vs. NL/NG $p<0.001$ ) (Table II, Figure 2). We were expecting that patients with hyperlipidemia and hyperglycemia (two factors responsible for platelet activation) would present higher concentrations of sP-selectin than patients with a single risk factor. Nevertheless, we did not observe intensification of platelet activity in the HL/HG group.

Furthermore, we noted a higher percentage of PDMPs in all groups of stroke patients compared to CS ( $p<0.001)$, but we did not observe an influence of $\mathrm{HL}$ or $\mathrm{HG}$ on the percentage of circulating PDMPs (Table II, Figure 3).

The result of our study showed a significantly lower percentage of CD62(+) platelets and lower CD62P expression on resting and thrombin-activated platelets in all groups of stroke patients compared with the controls (Table II, Figure 4). An

Table II. Table presents medians of the percentages of CD62P-positive platelets (\%CD62) and the median fluorescence intensity of CD62P expression (med CD62) on resting and thrombin-activated platelets, percentage of platelet-derived microparticles (PDMPs) and soluble P-selectin concentration in blood of the study patients and control subjects

\begin{tabular}{|c|c|c|c|c|c|}
\hline \multirow[t]{2}{*}{ Study parameters } & \multicolumn{5}{|c|}{ Median } \\
\hline & Control & NL/NG & NL/HG & HL/NG & HL/HG \\
\hline$\%$ CD62 before thrombin & 2.40 & $1.20^{*}$ & $1.40^{\star}$ & $1.05^{*}$ & 1.35 \\
\hline \%CD62 after thrombin & 28.45 & $8.00^{* *}$ & $3.80^{\star *}$ & $14.35^{\star}$ & $14.40^{*}$ \\
\hline Med CD62 before thrombin & 6.34 & $5.34^{*}$ & $4.75^{\star}$ & $4.99^{*}$ & $4.90^{\star *}$ \\
\hline Med CD62 after thrombin & 29.48 & $17.97^{\star *}$ & $14.70^{\star *}$ & $20.60^{\star}$ & $23.88^{\star *}$ \\
\hline PDMPs [\%] & 8.30 & $12.80^{\star *}$ & $12.55^{\star \star}$ & $13.20^{\star *}$ & $13.25^{\star \star}$ \\
\hline sP-selectin [ng/ml] & 70.75 & $107.66^{\star *}$ & $128.93^{\star * \star \#}$ & $124.65^{\star * * \# \#}$ & $134.11^{\star * * \# \#}$ \\
\hline
\end{tabular}

NL/NG - normolipidemic/normoglycemic group, NL/HG - normolipidemic/hyperglycemic group, HL/NG - hyperlipidemic/normoglycemic group, HL/HG - hyperlipidemic/hyperglycemic group, CS - control subjects; ${ }^{*} p<0.05 \mathrm{vs}$. control, ${ }^{* *} p<0.001 \mathrm{vs}$. control, ${ }^{* * *} p<0.0001 \mathrm{vs}$.

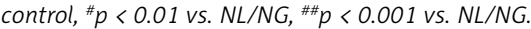




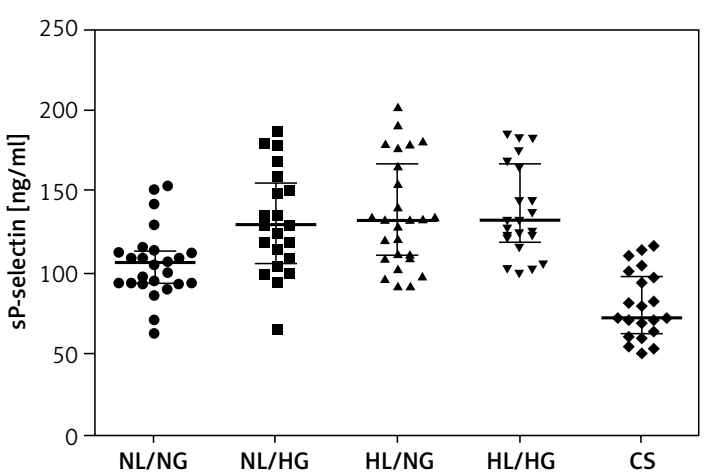

Figure 2. sP-selectin serum concentration was significantly higher in all stroke patients compared to control subjects (NL/NG vs. CS $p<0.001$ and $\mathrm{NL} / \mathrm{HG}, \mathrm{HL} / \mathrm{NG}, \mathrm{HL} / \mathrm{HG}$ vs. CS $p<0.0001)$ and in patients with metabolic disorders compared to $\mathrm{NL} / \mathrm{NG}$ stroke patients (NL/HG vs. NL/NG $p<0.01$; $\mathrm{HL} / \mathrm{NG}, \mathrm{HL} / \mathrm{HG}$ vs. NL/NG $p<0.001)$

$N L / N G$ - normolipidemic/normoglycemic group, NL/HG - normolipidemic/hyperglycemic group, HL/NG - hyperlipidemic/normoglycemic group, HL/HG - hyperlipidemic/hyperglycemic group, CS - control subjects.
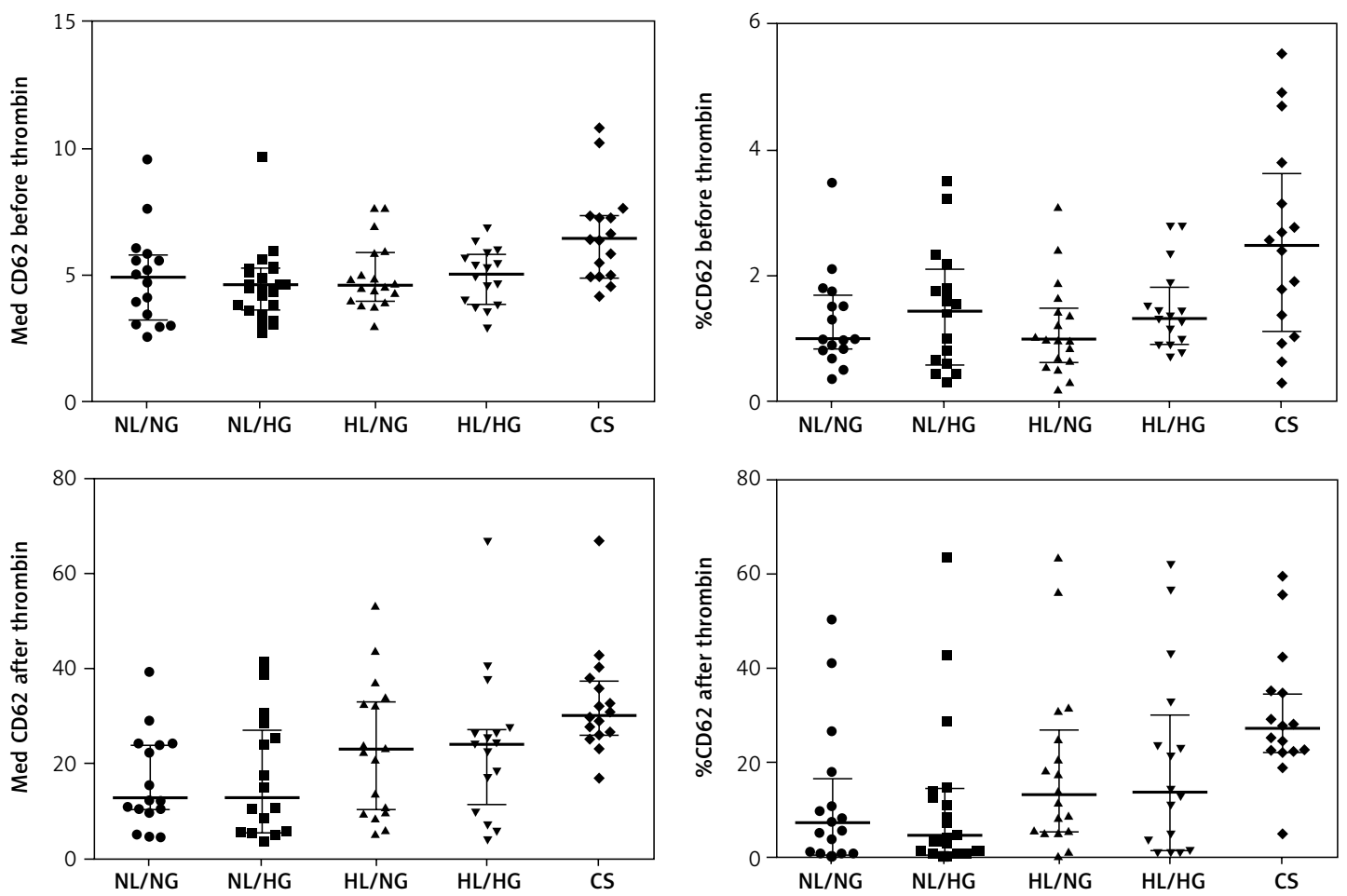

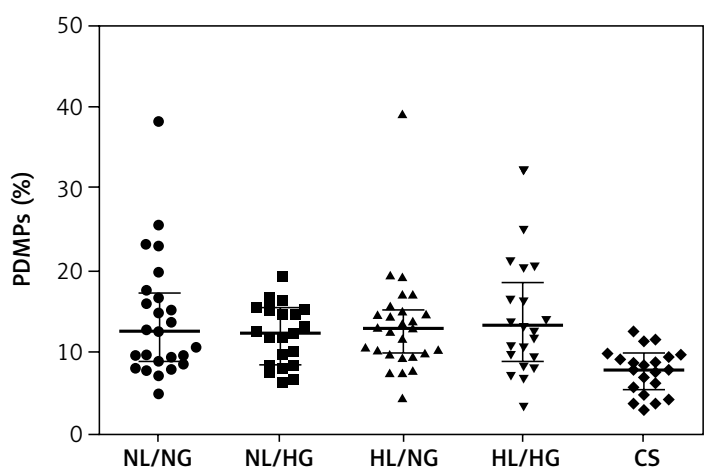

Figure 3. Percentage of PDMPs was significantly higher in all groups of stroke patients compared to CS, $p<0.001$

NL/NG - normolipidemic/normoglycemic group, NL/HG - normolipidemic/hyperglycemic group, HL/NG - hyperlipidemic/normoglycemic group, HL/HG - hyperlipidemic/hyperglycemic group, CS - control subjects.

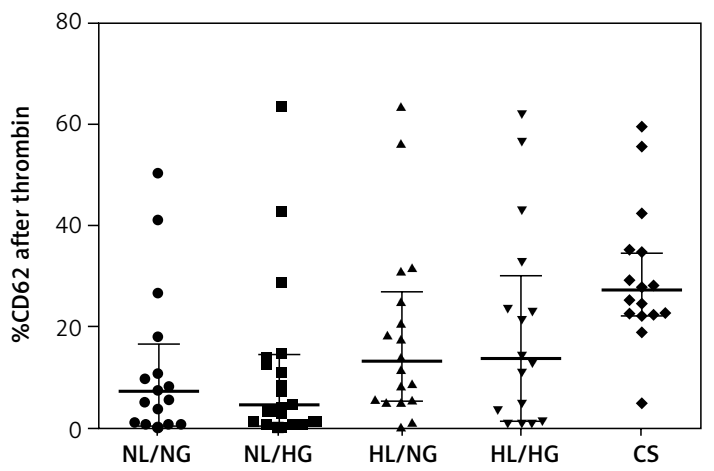

Figure 4. Median of fluorescence CD62P (med CD62) and percentage of platelets CD62P+ (\%CD62) before and after stimulation by thrombin were significantly lower in all groups of stroke patients compared with CS

NL/NG - normolipidemic/normoglycemic group, NL/HG - normolipidemic/hyperglycemic group, HL/NG - hyperlipidemic/ normoglycemic group, HL/HG - hyperlipidemic/hyperglycemic group, CS - control subjects.

influence of metabolic disorders (hyperlipidemia and hyperglycemia) on the expression of CD62P on resting and thrombin-activated platelets was not found. There were no significant differences in the median of CD62P fluorescence and percentage of $\mathrm{CD62} \mathrm{P}(+)$ platelets before and after thrombin activation between the stroke groups (Table II, Figure 4). 
concentration, platelet P-selectin expression and PDMPs. The results of our study demonstrate the significant influence of hyperlipidemia and hyperglycemia on SP-selectin concentration in patients with ischemic stroke. We observed a significant increase in SP-selectin plasma concentration in stroke patients with hyperlipidemia and hyperglycemia compared to normolipidemic and normoglycemic stroke patients. We also found a significant positive correlation between LDL, total cholester$\mathrm{ol}, \mathrm{HbA}_{1 c}$ level and sP-selectin concentration. This observation confirms the significant influence of hyperlipidemia and hyperglycemia on endothelium and platelet activation. We cannot be sure whether the higher sP-selectin levels observed in our study were released from the platelet $\alpha$-granule membrane or from the Weibel-Palade body membrane of endothelial cells.

Circulating atherogenic lipoproteins have been shown to activate platelets, and hence the changes in platelet function may also be secondary to the vascular disease caused by the dyslipidemia [4]. In accordance with our results, Ferroni et al. [20] and Davi et al. [21] confirmed the relationship between increased LDL concentration and plasma SP-selectin level. They concluded that hyperlipidemia is associated with an increase of plasma sP-selectin level. Other studies have also confirmed the influence of hyperlipidemia on platelet activity [22, 23]. Data of these studies showed a significant positive correlation between platelet P-selectin expression and increased LDL level. These studies confirmed the hypothesis that hypercholesterolemia may be associated with persistent platelet activation. On the other hand, high cholesterol level is frequently associated with development of atherosclerosis. It has been observed that P-selectin expression is increased in endothelial cells overlying atherosclerotic plaques [24]. Thus high P-selectin plasma levels may also be an index of the presence of vascular lesions [21].

Hyperreactive platelets are also the major determinants of the prothrombotic state in diabetic patients [25]. The causes of the enhanced platelet activation in diabetes are multifactorial. Platelet abnormalities include altered adhesion, aggregation and hypersensitivity to agonists. Of note, glycosylation and oxidation caused by hyperglycemia increase atherogenicity of circulating LDL. All these mechanisms may potentially contribute to platelet hyperfunction and hypersensitivity [26, 27]. Changes in the cell membrane dynamics observed in diabetes result in altered exposure of surface membrane receptors [28]. P-selectin has been identified as the more important adhesion molecule. A previous study by Nomura et al. [29] confirmed these findings. They demonstrated an elevated soluble P-selectin level in hyperlipidemic patients with type 2 diabetes, and an association with increased oxidized LDL. We also observed a significantly higher concentration of sP-selectin in all groups of stroke patients with hyperglycemia. It is important to add that the strongest positive correlation was observed between hyperglycemia $\left(\mathrm{HbA}_{1 \mathrm{c}}\right)$ and sP-selectin levels. This observation emphasizes the leading role of hyperglycemia in atherothrombosis progression. We could expect the intensification of platelet activity and higher sP-selectin concentration in patients with hyperlipidemia and hyperglycemia. Nevertheless, we did not observe additional effects of hyperlipidemia and hyperglycemia on platelet activity markers.

The interesting role of sP-selectin is stimulating the release of procoagulant microparticles, inducing a procoagulant state [30, 31]. A number of studies have established a clear relationship with thrombosis development in cerebrovascular ischemic diseases [32, 33]. Nomura et al. [34] reported significantly higher PDMP blood concentrations in diabetic patients with increased LDL levels than in diabetic patients with low LDL levels. This finding suggests that PDMPs may participate in the development and progression of atherosclerosis. On the other hand, our data indicated an increase of PDMP level in all stroke patients, but without an influence of hyperlipidemia or hyperglycemia on PDMP concentration. These results are concordant with the findings of Cherian et al. [35]. They observed significantly higher PDMP levels within 7 days of stroke onset in comparison to controls. Elevated levels of PDMPs observed in stroke patients may confirm the important role of PDMPs in coagulation and their thrombogenic potential. It is also possible that the local generation of PDMPs in atherosclerotic arteries may promote arterial occlusion [36].

On the other hand, an increased PDMP level in the convalescent phase after ischemic stroke was confirmed by other studies [32, 33]. Thus, it is currently difficult to determine whether the origin of the PDMPs results from acute ischemic stimulation or it is the consequence of chronic platelet activation.

Many studies have demonstrated the increase of platelet $\mathrm{P}$-selectin expression in ischemic stroke [37-42], reflecting platelet hyperactivity during the acute phase of ischemia. However, our study did not demonstrate higher expression of platelet P-selectin during the first 7 days of stroke. We observed significantly lower platelet P-selectin expression in all stroke patients compared to control subjects. One explanation of this finding may be the fact that in previous studies the time point of P-selectin assessment in acute stroke was different. In some studies the assessment of platelets was carried out relatively late (after 4 weeks) [42], while in others platelet activation was assessed 
within $24 \mathrm{~h}$ from the onset of stroke [37, 39]. However, our data are supported by the study of Marquardt et al. [40]. They observed an initial increase in P-selectin platelet expression only on the first day, then platelet P-selectin expression declined over time after the stroke, and the difference between the stroke group and controls was no longer significant on day 14 after the stroke. The rapid decrease in P-selectin expression may be caused by the shedding from the cell surface to the plasma pool. This hypothesis may explain our results, because we observed lower expression of platelet P-selectin but a significantly higher soluble P-selectin level in stroke patients compared to CS. These results may be evidence of rapid platelet activation in the acute phase of stroke. Thus, in view of our findings and the results of previous studies, we suggest that sP-selectin is a better marker of platelet activation than platelet P-selectin. However, we should remember that the origin of sP-selectin in blood may reflect the release of this adhesion molecule from activated platelets and also from damaged endothelial cells [43]. So, additional assessment of an endothelial injury marker could be helpful in this analysis.

It is noteworthy that we also observed decreased platelet reactivity in stroke patients. We observed lower platelet P-selectin expression after thrombin activation in all stroke patients compared to CS. This finding may be due to exhaustion of P-selectin stores in alpha granules, and this may partly explain the early hemorrhagic complication in the acute phase of stroke. Our observation is consistent with the results of Lukasik et al. [44]. They also confirmed lower surface expression of P-selectin under thrombin receptor activating peptide (TRAP) stimulation in patients with acute stroke.

In conclusion, plasma sP-selectin is a good marker reflecting the significant influence of metabolic disorders such as hyperglycemia and hyperlipidemia on vascular lesions and platelet activation in stroke patients. Metabolic disturbances exert an equal stimulatory effect on tested platelet markers but with no synergistic action. However, the results of our study point to the leading role of hyperglycemia in the atherothrombotic process. The enhanced SP-selectin concentration in atherosclerotic ischemic stroke is the consequence of atherogenic and platelets' procoagulant properties of oxidized LDL and hyperglycemia, but an increased level of this molecule may also reveal the effect of acute ischemic events [36]. Further studies are required to identify the main source of plasma P-selectin origin in patients with metabolic disturbances, because the increased sP-selectin level mediates a procoagulant state, potentially exacerbating the prothrombotic predisposition associated with hyperlipidemia and hyperglyce- mia. Thus, sP-selectin is a good target for further research focusing on prevention of ischemic cerebrovascular diseases.

\section{Acknowledgments}

We would like to thank Mrs. Anna Augustyniak for her technical assistance. The study was supported by a grant from the Medical University of Lodz, Poland (grant no. 502-03/5-062-01/502-54-009).

\section{Conflict of interest}

The authors declare no conflict of interest.

\section{References}

1. Ahmad SS, London FS, Walsh PN. The assembly of the factor $\mathrm{X}$ activating complex on activated human platelets. J Thromb Haemost 2003; 1: 48-59.

2. Boncler M, Gresner P, Nocun M, et al. Elevated cholesterol reduces acetylsalicylic acid-mediated platelet acetylation. Biochim Biophys Acta 2007; 1770: 1651-9.

3. Sahebkar A, Serban C, Ursoniu S, et al. Lipid and Blood Pressure Meta-analysis Collaboration (LBPMC) Group. The impact of statin therapy on plasma levels of von Willebrand factor antigen. Systematic review and meta-analysis of randomised placebo-controlled trials. Thromb Haemost 2016; 115: 520-32.

4. Milionis J, Elisaf MS, Mikhailidis DP. Platelet function and lipid-lowering interventions. Platelets 1999; 10: 357-67.

5. Aronow WS. Lipid-lowering therapy in older persons. Arch Med Sci 2015; 11: 43-56.

6. Creager MA, Lüscher TF, Cosentino F, Beckman JA. Diabetes and vascular disease: pathophysiology, clinical consequences, and medical therapy: part I. Circulation 2003; 108: 1527-32.

7. Osende JI, Badimon JJ, Fuster V, et al. Blood thrombogenicity in type 2 diabetes mellitus patients is associated with glycemic control. J Am Coll Cardiol 2001; 38: 1307-12.

8. Takada Y, Urano T, Watanabe I, Taminato A, Yoshimi T, Takada A. Changes in fibrinolytic parameters in male patients with type 2 (non-insulin-dependent) diabetes mellitus. Thromb Res 1993; 71: 405-15.

9. Watala C. Blood platelet reactivity and its pharmacological modulation in (people with) diabetes mellitus. Curr Pharmaceut Design 2005; 11: 2331-65.

10. Ferreiro JL, Gómez-Hospital JA, Angiolillo DJ. Platelet abnormalities in diabetes mellitus. Diab Vasc Dis Res 2010; 7: 251-9.

11. Blann AD, Nadar SK, Lip GY. The adhesion molecule P-selectin and cardiovascular disease. Eur Heart J 2003; 24: 2166-79.

12. Michelson AD, Barnard MR, Hechtman HB, et al. In vivo tracking of platelets: circulating degranulated platelets rapidly lose surface $P$ selectin but continue to circulate and function. Proc Natl Acad Sci USA 1996; 93: 11877-82.

13. Burger PC, Wagner DD. Platelet P-selectin facilitates atherosclerotic lesion development. Blood 2003; 101: 2661-6.

14. Andre P, Hartwell D, Hrachovinova I, Saffaripour S, Wagner DD. Pro-coagulant state resulting from high levels of soluble P-selectin in blood. Proc Natl Acad Sci USA 2000; 97: $13835-40$. 
15. Nomura S. Function and clinical significance of platelet-derived microparticles. Int J Hematol 2001; 74: 397-404.

16. Adams Jr HP, Bendixen BH, Kappelle LJ, et al. Classification of subtype of acute ischemic stroke. Definitions for use in a multicenter clinical trial. TOAST. Trial of Org 10172 in Acute Stroke Treatment. Stroke 1993; 24: 35-41.

17. Catapano AL, Reiner Z, De Backer G, et al.; European Society of Cardiology (ESC); European Atherosclerosis Society (EAS). ESC/EAS Guidelines for the management of dyslipidaemias The Task Force for the management of dyslipidaemias of the European Society of Cardiology (ESC) and the European Atherosclerosis Society (EAS). Atherosclerosis 2011; 217: 3-46.

18. Authors/Task Force Members, Rydén L, Grant PJ, Anker SD, et al. ESC Guidelines on diabetes, pre-diabetes, and cardiovascular diseases developed in collaboration with the EASD: the Task Force on diabetes, pre-diabetes, and cardiovascular diseases of the European Society of Cardiology (ESC) and developed in collaboration with the European Association for the Study of Diabetes (EASD). Eur Heart J 2013; 34: 3035-87. Erratum in Eur Heart J 2014; 35: 1824.

19. Krishnaveni P, Gowda V. Assessing the validity of Friedewald's formula and Anandraja's formula for serum LDL-cholesterol calculation. J Clin Diagn Res 2015; 9: BC01-4.

20. Ferroni P, Basili S, Vieri M, et al. Soluble Pselectin and proinflammatory cytokines in patients with polygenic type Ila hypercholesterolemia. Haemostasis 1999; 29: 277-85.

21. Davi G, Romano M, Mezzetti A, et al. Increased levels of soluble P-selectin in hypercholesterolemic patients. Circulation 1998; 97: 953-7.

22. Gebuhrer V, Murphy JF, Bordet JC, Reck MP, McGregor JL. Oxidized low-density lipoprotein induces the expression of P-selectin (GMP 140/PADGEM/CD62) on human endothelial cells. Biochem J 1995; 306: 293-8.

23. Sener A, Ozsavci D, Oba R, Demirel GY, Uras F, Yardimci KT. Do platelet apoptosis, activation, aggregation, lipid peroxidation and platelet-leukocyte aggregate formation occur simultaneously in hyperlipidemia? Clin Biochem 2005; 38: 1081-7.

24. Johnson-Tidey RR, McGregor JL, Taylor PR, Poston RN. Increase in the adhesion molecule P-selectin in endothelium overlying atherosclerotic plaques: coexpression with intercellular adhesion molecule-1. Am J Pathol 1994; 144: 952-61.

25. Carr ME. Diabetes mellitus: a hypercoagulable state. J Diabetes Complications 2001; 15: 44-54.

26. Lyons TJ. Glycation and oxidation: a role in the pathogenesis of atherosclerosis. Am J Cardiol 1993; 71: 26-31.

27. Ferretti G, Rabini RA, Bacchetti T, et al. Glycated low density lipoproteins modify platelet properties: a compositional and functional study. J Clin Endocrinol Metab 2002; 87: 2180-4

28. Watala C, Gwozdzinski K, Pluskota E, et al. Diabetes mellitus alters the effect of peptide and protein ligands on membrane fluidity of blood platelets. Thromb Haemost 1996; 75: 147-53.

29. Nomura S, Kanazawa S, Fukuhara S. Effects of eicosapentaenoic acid on platelet activation markers and cell adhesion molecules in hyperlipidemic patients with type 2 diabetes mellitus. J Diabetes Complications 2003; 17: 153-9.

30. Andre P, Hartwell D, Hrachovinova I, Saffaripour S, Wagner DD. Pro-coagulant state resulting from high levels of soluble P-selectin in blood. Proc Natl Acad Sci USA 2000; 97: $13835-40$
31. Nomura S, Inami N, Iwasaka T, Liu Y. Platelet activation markers, microparticles and soluble adhesion molecules are elevated in patients with arteriosclerosis obliterans: therapeutic effects by cilostazol and potentiation by dipyridamole. Platelets 2004; 15: 167-72.

32. Pawelczyk M, Baj Z, Chmielewski H, Kaczorowska B, Klimek A. The influence of hyperlipidemia on platelet activity markers in patients after ischemic stroke. Cerebrovasc Dis 2009; 27: 131-7.

33. Serebruany VL, Malinin Al, Ziai W, et al. Effects of clopidogrel and aspirin in combination versus aspirin alone on platelet activation and major receptor expression in patients after recent ischemic stroke: for the Plavix Use for Treatment of Stroke (PLUTO-Stroke) trial. Stroke 2005; 36: 2289-92.

34. Nomura S, Suzuki M, Katsura K, et al. Platelet-derived microparticles may influence the development of atherosclerosis in diabetes mellitus. Atherosclerosis 1995; 116: 235-40.

35. Cherian P, Hankey GJ, Eikelboom JW, et al. Endothelial and platelet activation in acute ischemic stroke and its etiological subtypes. Stroke 2003; 34: 2132-7.

36. McGregor L, Martin J, McGregor JL. Platelet-leukocyte aggregates and derived microparticles in inflammation, vascular remodeling and thrombosis. Front Biosci 2006; 1: 830-7.

37. Htun P, Fateh-Moghadam S, Tomandl B, et al. Course of platelet activation and platelet-leukocyte interaction in cerebrovascular ischemia. Stroke 2006; 37: 2283-7.

38. Cha JK, Jeong MH, Jang JY, et al. Serial measurements of CD63, P-selectin and CD40 ligand on platelets in atherosclerotic ischemic stroke. Cerebrovasc Dis 2003; 16 376-382.

39. Grau, AJ, Ruf A, Vogt A, et al. Increased fraction of circu lating activated platelets in acute and previous cerebrovascular ischemia. Thromb Haemost 1998; 80: 298-301.

40. Marquardt L, Ruf, A, Mansmann U, et al. Course of platelet activation markers after ischemic stroke. Stroke 2002; 33: 2570-4

41. Garlichs CD, Kozina S, Fateh-Moghadam S, et al. Upregulation of CD40-CD40 ligand (CD154) in patients with acute cerebral ischemia. Stroke 2003; 34: 1412-8.

42. McCabe DJ, Harrison P, Mackie IJ, et al. Platelet degranulation and monocyte-platelet complex formation are increased in the acute and convalescent phases after ischaemic stroke or transient ischaemic attack. Br J Haematol 2004; 125: 777-87.

43. Michelson AD, Barnard MR, Hechtman HB, et al. In vivo tracking of platelets: circulating degranulated platelets rapidly lose surface Pselectin but continue to circulate and function. Proc Natl Acad Sci USA 1996; 93: 11877-82.

44. Lukasik M, Dworacki G, Michalak S, et al. Aspirin treatment influences platelet-related inflammatory biomarkers in healthy individuals but not in acute stroke patients. Thromb Res 2011; 128: 73-80. 